\title{
Breast Cancer Metastasis Masquerading as the Great Masquerader: Sebaceous Cell Carcinoma
}

\author{
Jonathan Martin ${ }^{a} \quad$ Maxwell A. Fung ${ }^{b} \quad$ Lily Koo Lin ${ }^{a}$ \\ ${ }^{a}$ Department of Ophthalmology and Vision Science, University of California Davis Health \\ System, Sacramento, CA, USA; ${ }^{b}$ Department of Dermatology, University of California Davis \\ Health System, Sacramento, CA, USA
}

\section{Keywords}

Breast carcinoma · Eyelid metastasis · Madarosis · Eyelid margin thickening

\begin{abstract}
Objective: We describe the case of a patient with metastatic breast cancer who presented with eyelid margin thickening and madarosis more suggestive of sebaceous cell carcinoma than metastatic disease. Histopathology confirmed metastatic breast adenocarcinoma. Case Report: A 59-year-old woman with a known history of metastatic breast carcinoma actively enrolled in a clinical trial presented with a thickened right upper eyelid margin with madarosis and without ulceration. Although the possibility of metastasis was considered, a biopsy was performed to ensure the patient did not have a primary eyelid malignancy such as sebaceous cell carcinoma given her immunocompromised state. Histopathology revealed metastatic breast adenocarcinoma. Conclusions: To the authors' best knowledge, metastatic breast carcinoma presenting as eyelid margin thickening without ulceration has not previously been reported. Eyelid metastasis is rare, and this patient's clinical presentation was found to be unusual as well. It is important to establish metastatic disease even in a small focus such as the eyelid, as it may alter disease management.
\end{abstract}




\section{Case Reports in Oncology}

Martin et al.: Breast Cancer Metastasis Masquerading as the Great Masquerader: Sebaceous Cell Carcinoma

\section{Introduction}

Sebaceous cell carcinoma is often heralded as the "great masquerader" with its variability in presentation, and deceptively benign-appearing eyelid features. It arises from sebaceous glands, commonly from the meibomian glands in the tarsus, and thus can present as thickening or yellow appearance of the tarsus. Sebaceous cell carcinoma is also known for its pagetoid spread, with malignant cells separate from the tumor focus. We present a rare case of a right upper eyelid breast cancer metastasis with shared features of sebaceous cell carcinoma both clinically and on histopathology.

\section{Case Report}

A 59-year-old female was diagnosed in 2008 with stage I, grade 2, invasive ductal carcinoma, estrogen receptor and progesterone receptor positive, Her2/neu nonamplified, of the right breast. Sentinel lymph node biopsy was negative. She was treated with lumpectomy, adjuvant radiation with a brief course of tamoxifen, then anastrozole, with both medications self-discontinued due to toxicities.

In 2013, a screening mammogram revealed bilateral abnormalities, and bilateral biopsies revealed invasive ductal carcinoma, estrogen and progesterone receptor positive. She underwent bilateral mastectomy and was found to have extranodal disease. Positron emission tomography scan showed diffuse metastasis to the bones, spine, and scalp with biopsy confirmation of metastatic disease. She was treated with numerous chemotherapeutics and radiation therapy and enrolled in a clinical trial.

In November 2015, she noted a thickening of the right upper eyelid and missing eyelashes, and in February 2016, the patient was seen in the Oculoplastics Service. She denied any pain from the area and only noted it when she went to apply mascara.

Her examination at the time of consultation was best-corrected visual acuity of 20/40 in the right eye and 20/30 in the left eye. Pupils were without relative afferent pupillary defect. Her extraocular motility was full. Her examination was notable for an area of central madarosis and lid margin thickening of the right upper lid resulting in lid margin eversion; there was no ulceration of the lesion (Fig. 1a).

A biopsy was performed the same day under local anesthesia in the office. Biopsy of the right upper eyelid showed infiltrative aggregates of tumor cells with scant cytoplasm in the dermis, with limited involvement of the epidermis. Cytologic detail was obscured by crush artifact, but there was no visible cytoplasmic vacuolization to suggest sebaceous or other glandular differentiation (Fig. 1b). Single file growth was evident. The tumor cells were immunopositive for cytokeratin 7, Cam5.2 (contains cytokeratins 7/8), and epithelial membrane antigen (Fig. 1c).

After her positive eyelid biopsy, it was considered as evidence of disease progression. She discontinued the clinical trial and was started on fulvestrant with palbociclib. She has had mixed response by positron emission tomography and tumor markers but was overall stable. 


\section{Case Reports in Oncology}

Martin et al:: Breast Cancer Metastasis Masquerading as the Great Masquerader: Sebaceous Cell Carcinoma

\section{Discussion}

Eyelid metastasis is rare, and in multiple case series accounts for $<1 \%$ of malignant eyelid lesions [1, 2], with the vast majority representing basal cell carcinoma $(80.4 \%)$, and smaller numbers of squamous cell carcinoma (7\%), malignant melanoma (5.1\%), and sebaceous carcinoma (3.3\%) [1]. Further, ocular metastatic disease is much more common with only $1.1 \%$ of patients having eyelid metastasis in a review by Bianciotto et al. [3]. However, of the small number of metastases to the eyelids, breast carcinoma represented 40,35 , and $15 \%$ of the primary tumor sites in 3 case series [3-5].

Although primary carcinoma of the eyelid is far more common than metastatic eyelid involvement, metastatic breast carcinoma may be among the more common causes of metastasis in the eyelid. In this case, the presence of pagetoid intraepidermal growth represents an overlapping feature between sebaceous carcinoma and epidermotropic metastasis. However, the presence of cutaneous adenocarcinoma in a patient with a preexisting history of breast carcinoma represents presumptive evidence of metastatic breast cancer, most typically arising on the head/neck or upper torso. Histopathologically, the tumor was poorly differentiated and exhibited infiltrative single file growth and scant cytoplasm, characteristic of metastatic carcinoma and not typical of sebaceous carcinoma, including poorly differentiated examples.

While the overwhelming burden of malignant eyelid tumors represents primary tumors, it is important to consider metastatic disease, particularly in patients with a known cancer diagnosis, as establishing a metastasis can alter disease management.

The study adhered to the principles of the Declaration of Helsinki and was exempted from the Institutional Review Board of the University of California Davis Health System.

\section{Statement of Ethics}

Verbal consent was obtained to publish case details including the clinical photograph.

\section{Disclosure Statement}

The authors declare that they have no financial disclosures.

\section{References}

1 Aurora AL, Blodi FC: Lesions of the eyelids: a clinicopathological study. Surv Ophthalmol 1970;15:94104.

Arnold AC, Bullock JD, Foos RY: Metastatic eyelid carcinoma. Ophthalmology 1985;92:114-119.

Bianciotto C, Demirci H, Shields CL, et al: Metastatic tumors to the eyelid: report of 20 cases and review of the literature. Arch Ophthalmol 2009;127:999-1005.

4 Riley FC: Metastatic tumors of the eyelids. Am J Ophthalmol 1970;69:259-264.

-5 Mansour AM, Hidayat AA: Metastatic eyelid disease. Ophthalmology 1987;94:667-670. 


\section{Case Reports in Oncology}
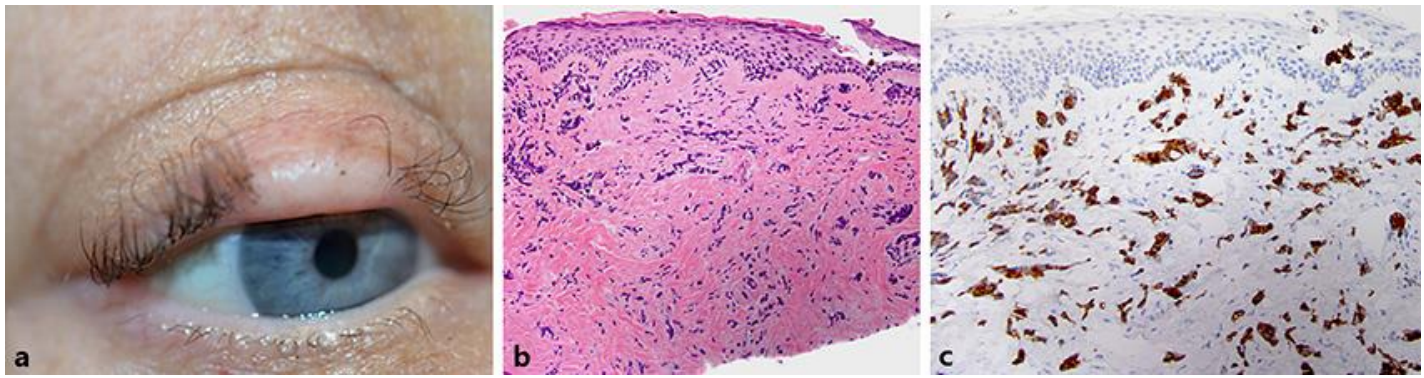

Fig. 1. a External photograph to demonstrate the clinical appearance of the right upper eyelid lesion. b Epidermotropic metastatic breast carcinoma. Infiltrative aggregates of tumor cells with scant cytoplasm in the dermis, with limited involvement of the epidermis. Cytologic detail is obscured by crush artifact. HE. $\times 200$. c Epidermotropic metastatic breast carcinoma. The dermal and intraepidermal tumor cells were diffusely immunopositive for cytokeratin 7 . CK7 $\times 200$. 